\title{
Computerized ultrasound image analysis for noninvasive evaluation of hepatic steatosis.
}

\author{
Georgiana Nagy $^{1 *}$, Mihai Munteanu ${ }^{1 *}$, Mihaela Gordan², Romeo Chira ${ }^{1}$, Mihaela Iancu ${ }^{3}$, \\ Doinita Crisan ${ }^{4}$, Petru Adrian Mircea ${ }^{1}$
}

${ }^{1} 1^{\text {st }}$ Department of Internal Medicine, "Iuliu Hatieganu" University of Medicine and Pharmacy ${ }^{2}$ Technical University, ${ }^{3}$, Biostatistics Department, "Iuliu Hatieganu" University of Medicine and Pharmacy, ${ }^{4}$ Pathology Department, "Iuliu Hatieganu" University of Medicine and Pharmacy, Cluj-Napoca, Romania

\begin{abstract}
Objective: To assess the diagnostic value of a particular set of local intensity parameters extracted from ultrasound liver images in conjunction with support vector machine (SVM) classifiers for liver steatosis grading in respect to the "gold standard" provided by liver biopsy. Material and methods: We prospectively enrolled in the study 228 patients with chronic hepatopathies. All the patients underwent liver biopsy and abdominal ultrasound examination. For quantitative ultrasound assessment of liver steatosis, an image analysis software was developed, which extracts three local intensity parameters from regions of interest (ROI) in the ultrasound section and analyzes their depth variation: the coefficient of variation of luminance $(\mathrm{CVL})$, the median luminance $\left(\mathrm{m}_{1}\right)$, and the hepato-splenic attenuation index (HSAI). For steatosis grading, SVM classifiers were trained on the input feature spaces provided by the above mentioned parameters. The statistical significance of the steatosis grading was assessed on a significant test set using SVM classifiers, in terms of sensibility, specificity and through the ROC curves. Results: A cut-off value of 0.362 of the CVL of the liver performed the liver steatosis grading with an accuracy of $89.17 \%(\mathrm{p}<0.0001)$. A cut-off value of 0.27 of the HSAI performed the prediction of the moderate-severe liver steatosis with an accuracy of $87 \%$. Conclusions: The proposed computer analysis method of ultrasound images proved innovative and useful for the initial non-invasive assessment and grading of liver steatosis, with an additional advantage of reduced computational complexity and accessibility. The CVL provided a very good accuracy $(89.17 \%)$ for an AUROC of 0.923 for the classification of liver steatosis in two severity categories (mild versus moderate-severe).
\end{abstract}

Keywords: quantitative liver ultrasound, computer assisted image analysis, steatosis, chronic hepatopathies

\section{Introduction}

Hepatic steatosis (HS) is an anatomo-clinical entity frequently encountered in most diffuse chronic liver diseases (viral, nonalcoholic fatty liver, alcoholic etc.). Nonalcoholic fatty liver disease (NAFLD) is currently the most common cause of chronic liver diseases, affecting 1 billion people worldwide [1]; by 2030 it is expected

Received 02.09.2015 Accepted 01.10.2015

Med Ultrason

2015, Vol. 17, No 4, 431-436

Corresponding author: Georgiana Nagy

1st Department of Internal Medicine

3-5 Clinicilor street

400006 Cluj-Napoca, Romania

Phone: +40 724450307, Fax: +40264590899

E-mail: georgi_nagy@yahoo.com to become the main indication for liver transplantation [2]. Chronic viral hepatitis B (HBV) and C (HCV) are both associated with HS, with a prevalence of $14-70 \%$ for $\mathrm{HBV}$ and $62-76 \%$ for $\mathrm{HCV}$, especially in association with the HCV genotype 3a [3]. Though HS was earlier considered a benign condition, many recent studies indicate that fatty liver is more vulnerable to various aggressive factors, ultimately leading to inflammation, fibrosis, and cirrhosis [4]. Furthermore, in patients with HBV and $\mathrm{HCV}$, the presence of HS was associated with a progression of hepatic fibrosis (HF) and a lack of response to antiviral therapy [5-7].

In view of the above, the assessment and grading of HS severity in patients with diffuse chronic liver diseases is essential. The liver biopsy, despite its limitations and inconveniences (i.e., its invasive nature, risk of compli- 
cations, sampling errors) $[8,9]$, continues to represent the gold standard for the quantification, staging, and grading of HS. Considering these limitations and the non-reproducibility of the liver biopsy to monitor the evolution of chronic hepatopathies, alternative non-invasive reproducible methods to assess HS have been researched and developed [10].

One of the most frequently used imaging methods to assess HS is hepatic ultrasound (US), especially popular due to its accessibility, simplicity and non-ionizing character; furthermore, hepatic US has been shown to provide a rather good accuracy in the identification of the moderate-severe HS [11]. However, there are some important limitations of the hepatic US imaging procedure, such as: lack of differentiation between HS and steatohepatitis; inability to quantitatively assess the liver fat by simple visual examination; difficulty to accurately discriminate HS and HF, since the two conditions can result in a visually similar US pattern [12]. These limitations can be overcome by the means of US images computer analysis (USICA). To quantitatively describe HS, USICA uses either echogenicity features of the hepatic parenchyma, either ultrasound attenuation features or local texture features (extracted from local histograms of regions of interest in the US liver section). The first studies for USICA adopted the non-separable wavelet transform to discriminate between the states of normal, steatosis, and cirrhosis or extracted the first and second-order grayscale parameters from the liver ultrasound images and employed a Bayesian classifier for the optimal eigenvector selection to categorize the diffuse liver disease (including fatty liver) $[13,14]$. Intensity histogram, intensity co-occurrence matrix, or texture feature number could be useful and important ultrasound characteristics to identify liver disease and to differentiate between fatty and normal liver $[15,16]$. Lupsor et al, using the US attenuation coefficient calculated in the US image, predicted mild, respectively moderate/severe steatosis with an AUROC of 0.734 and 0.842 , respectively [17].

Most of the recent studies use the so-called sonographic hepato-renal index (SHRI) to differentiate between the HS and the non-fatty liver. Significant correlation between steatosis (evaluated by liver biopsy and MRI spectroscopy) and SHRI has been found in several studies. The sensitivity and specificity of SHRI varied in different studies between $81.4-92.7 \%$ and $54-93 \%$, respectively [18-22].

This study aims to explore a simple, low cost, and sensitive method for USICA that can detect and quantify liver fat content. The coefficient of variation of luminance (CVL), the local median intensity in the liver US section $\left(\mathrm{m}_{1}\right)$ and the hepato-splenic attenuation index
(HSAI) have been extracted as numerical features and used for the characterization of HS, in reference to the histopathological examination (as gold standard) on a cohort of patients with chronic hepatopathies.

\section{Material and methods}

Between years 2007-2010 we prospectively included in this study 228 subjects diagnosed with chronic diffuse viral hepatopathies C, B, and NAFLD. The study was approved by the Ethics Committee of the University of Medicine and Pharmacy of Cluj-Napoca and informed consent was obtained from all participants according to the World Medical Association Declaration of Helsinki. All the patients were subject to liver biopsy for the disease staging and HS quantification according to the Brunt score. The day before liver biopsy, each patient underwent abdominal ultrasound examination. The hepatic and splenic ultrasound sections were stored in a database used further by USICA. We mention that preliminary results were published before [23] and this study presents the final work.

\section{Liver histology assessment}

Percutaneous US guided liver biopsy was performed with a Tru-Cut needle (Bard Biopty-Gun) (16-gauge). A liver specimen of $13 \mathrm{~mm}$ with at least 16 portal tracts was considered adequate for evaluation. All biopsy specimens were examined by the same pathologist blinded to the USICA results. The extent of HS and the degree of HF were assessed histologically according to the Brunt and Metavir criteria, respectively [24].

\section{US examination and computerized image analysis}

For the US examination, we used a Megas-Esaote Biomedica Italia ultrasound machine with a convex transducer of 3.5 MHz. The hepatic and splenic US sections were taken by placing the transducer at the intercostal spaces IX-X; these sections were saved as bitmap image files in a database. All the US images were taken under the same settings of the ultrasound machine by the same examiner, the processing parameters being set to: $\mathrm{B} / \mathrm{M}$ Gain - maximum, the curve "Time Gain Compensation" minimum, the examination depth $-10 \mathrm{~cm}$, so that the pre/ post-processing curves (PST) are 2/4; the pulse repetition frequency (PRF) was set to $6.1 \mathrm{kHz}$.

For USICA we developed a Windows application which allowed the automatic localization of a ROI of $1 \mathrm{~cm} \times 1 \mathrm{~cm}(40 \times 40$ pixels $)$ at three different depths in the liver parenchyma $(0.5,2$, and $3.5 \mathrm{~cm}$ depth from the hepatic capsule) followed by a local feature extraction in each ROI from the ROI intensity histogram (fig 1).

The most relevant features in terms of the HS description were: the median of the luminance $\mathrm{m}_{1}$ (numerical 


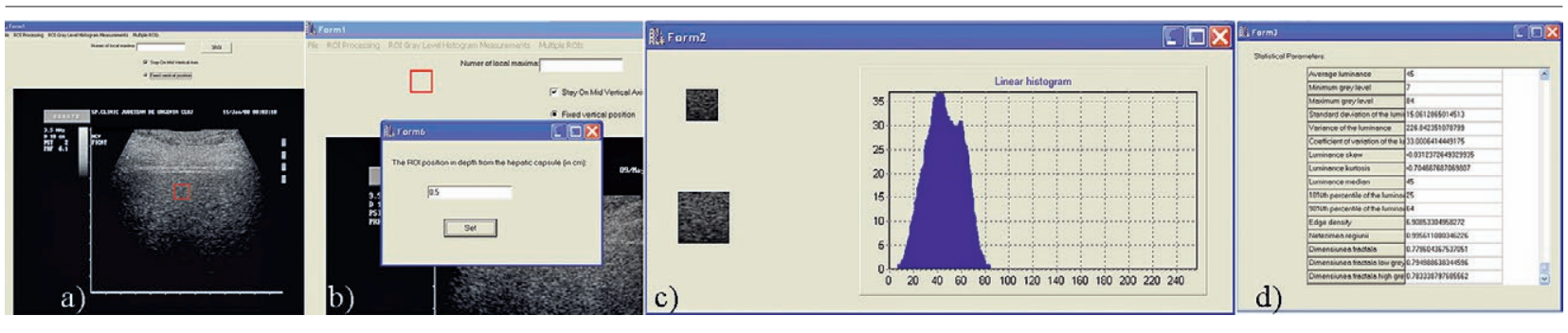

Fig 1. The software interfaces of the Windows application developed for USICA during different processing steps: a) after selecting and opening the US image to be analyzed; b) positioning the region of interest; c) displaying the smoothed linear grey levels (intensity) histogram of the region of interest; d) as a result of feature extraction, the numerical values of the echogenicity and local texture features used in the analysis are displayed.

descriptor of the median intensity in the ROI); the coefficient of variation of luminance, CVL, defined as the ratio of the standard deviation of the intensity in ROI to the mean intensity in ROI; and the hepato-splenic attenuation index (HSAI), defined as the difference between the local median intensity in a ROI of the liver ( $\mathrm{m}_{1}$ liver) and in a ROI of the spleen $\left(\mathrm{m}_{1}\right.$ spleen).

To assess the relevance of each feature, we employed support vector machine (SVM) classifiers to discriminate between the two HS severity classes (i.e., mild S0S1 versus moderate-severe S2-S3); we trained the SVM classifiers on a set of 108 patients (train group) and used the remaining 120 patients as the test group.

\section{Statistical analysis}

The statistical significance of the numerical features extracted to assess the HS severity was done by SVM classification, well known for its excellent generalization performance $[25,26]$. We used non-linear SVMs with RBF kernel - the most suitable for many medical data classification problems. The classification results were expressed in terms of accuracy, sensibility (Se), specificity $(\mathrm{Sp})$, positive, and negative predictive values (PPV, NPV). The diagnostic performance of each feature was also assessed by ROC curves; the cut-off value was set to maximize the sum of Se and Sp.

\section{Results}

From 228 examined patients, (mean age $44 \pm 11.38$ years, 117 male), 178 had chronic HCV infection. The histopathology reveals mild HS, S0-S1 (71.92\%) and moderate-severe HS, S2-S3 (28.08\%). In terms of HF, $44.73 \%$ of the patients were graded F2 and the others (67.09\%) with no/mild HF (F0-F2).

For USICA we grouped the patients with HS S0 and $\mathrm{S} 1$ into mild HS, and the patients with HS S2 and S3 into moderate-severe HS.

The overall accuracy in the discrimination of the two HS classes by each extracted feature is given in Table I.

Table I. The classification accuracy for hepatic steatosis grading in each individual feature space using non-linear support vector machine classifiers

\begin{tabular}{lll}
\hline Parameter & $\begin{array}{l}\text { Classification } \\
\text { accuracy } \\
\text { (train group) }\end{array}$ & $\begin{array}{l}\text { Classification } \\
\text { accuracy } \\
\text { (test group) }\end{array}$ \\
\hline Median luminance $(\mathrm{ml})$ & $100 \%$ & $88.34 \%$ \\
CVL & $100 \%$ & $89.17 \%$ \\
HSAI & $100 \%$ & $87 \%$ \\
\hline
\end{tabular}

CVL - coefficient of variation of luminance;

HSAI - hepato-splenic attenuation index

Table II. Statistical performance of parameters (coefficient of variation of luminance, median of luminance, hepato-splenic attenuation index) for hepatic steatosis grading (S0-S1vs S2-S3)

\begin{tabular}{llll}
\hline Parameter & CVL & ml & HSAI \\
\hline Cut-off value & 0.362 & 0.48 & 0.27 \\
Se (\%) [95\% CI] & $81.3[71.7-90.8]$ & $82.8[73.6-92.1]$ & $76.6[66.2-86.9]$ \\
Sp (\%) [95\% CI] & $89[84.9-93.8]$ & $75[68.4-81.6]$ & $80.5[74.4-86.6]$ \\
PPV(\%) [95\% CI] & $74.3[64-84.5]$ & $56.4[46.4-66.4]$ & $60.5[49.8-71.1]$ \\
PNV (\%) [95\% CI] & $92.4[88.3-96.5]$ & $91.8[87.1-96.4]$ & $89.8[84.9-94.7]$ \\
AUROC [95\% CI] & $0.923[0.87-0.97]$ & $0.871[0.81-0.93]$ & $0.864[0.8-0.92]$ \\
\hline
\end{tabular}

$\mathrm{CVL}=$ coefficient of variation of luminance, $\mathrm{ml}=$ median luminance, $\mathrm{HSAI}=$ hepato-splenic attenuation index, $\mathrm{Se}=\mathrm{sensitivity}, \mathrm{Sp}=\mathrm{speci}-$ ficity, $\mathrm{PPV}=$ predictive positive value, $\mathrm{PNV}=$ predictive negative value, AUROC = Area Under Receiver Operating Characteristic curve, $95 \% \mathrm{CI}=95 \%$ confidence interval 
CVL achieved a good accuracy of $89.17 \%$. At an optimal cut-off value of 0.362 for the study lot, CVL allowed the discrimination between mild and moderate-severe HS with $81.3 \%$ Se and $89 \% \mathrm{Sp}$, at AUROC close to the ideal value (0.923) (Table II).

The median liver luminance $\mathrm{m}_{1}$ has a slightly inferior performance to the CVL, with an accuracy of $88.34 \%$ (Table II). The optimal cut-off value for the prediction of moderate-severe HS was 0.48 , with Se and Sp of $82.8 \%$ and $75 \%$ for a good AUROC of 0.871 .

The new feature HSAI allows the discrimination of HS in the two severity classes with an accuracy of $86.7 \%$, Se of $76.6 \%$ and $\mathrm{Sp}$ of $80.5 \%$ for a good AUROC value of 0.864 (Table II).

The presence of significant HF $(F \geq 2)$ did not influence the staging of $\mathrm{HS}$ ( $\mathrm{p}=0.42$ for mild HS versus $\mathrm{p}=0.73$ for moderate-severe HS).

\section{Discussions}

In clinical practice, US is the first imaging method used for assessing chronic liver disease. The Se and Sp for HS detection using conventional B-mode US ranges between $60-94 \%$ and $88-95 \%$, is even lower $(80 \%$ and $55 \%$ respectively) when liver fat load is below $20 \%$ [27]. In contrast, USICA realized the objective quantification of US image characteristics of hepatic steatosis and showed significant advantages over traditional qualitative US. Using numerical features we were able to identify minimal changes in the liver echo pattern, which were impossible to distinguish by the naked eye.

In this study, the quantification of HS severity by USICA (using CVL, HSAI and $m_{1}$ parameters) was significantly correlated with histopathological evaluation results and was not influenced by the HF presence and severity. Accuracy of classification, Se and Sp of quantitative non-invasive diagnosis of fat load using the above mentioned parameters have been significantly higher than when using conventional US examination, which shows that USICA can be confidently used in clinical practice for evaluating more accurately the severity of fatty liver content .

A 0.362 cut-off value of CVL parameter for 0.923 AUROC has been useful to identify patients who have moderate-severe HS (>33\%), taking into account that this category of patients are at the greatest risk of progression to inflammation, fibrosis and cirrhosis. Identifying these patients at risk is important because they are subject to regular surveillance and additional, more costly or invasive, diagnosis methods (e.g. liver biopsy).

Statistical performances of CVL for quantifying HS are superior to those reported by existing similar stud- ies in the literature. Thus, using ultrasound attenuation coefficient (AC) (a parameter similar to CVL) Gaitini et al [16] achieved the prediction of severe HS with $88.9 \%$ accuracy, $90 \%$ Se and $88.5 \% \mathrm{Sp}$ on a lot of 24 patients evaluated by liver biopsy. Lupsor et al [17], at a -0.114 cut-off value of AC for 0.842 AUROC, predicted severe HS (>33\%) on a cohort of 189 patients with viral chronic $\mathrm{C}$ hepatitis assesed by liver biopsy, with $84.2 \%$ Se and $78.53 \%$ Sp. A recent study [22] using a quantitative ultrasound model based on a combination of AC and hepatorenal attenuation index (SHRI) has predicted moderate HS with $81.4 \%$ Se and $100 \% \mathrm{Sp}$, but the assesment of fat load was performed by MRI spectroscopy as the gold standard. It is known that the accuracy of MRI spectroscopy to assess HS is lower than histopathological evaluation; in addition it does not allow HS classification by severity grade. Liver biopsy, despite its inconveniencies, remains the reference method for HS severity grading [27].

The novelty in our study as compared with existing studies on USICA is the use, for the first time, of HSAI for the US quantitative assessment of HS. HSAI has the advantage of using the splenic parenchyma median intensity of the same patient as a reference knowing that both parenchymal tissues (liver and spleen) have similar echo features (echogenicity and texture) but the spleen does not accumulate fat. Furthermore, anatomically, the spleen is located at the same depth as the liver, compared with the thoracic wall, thus eliminating possible error factors (deep beam attenuation of ultrasound) linked to crossing /absorption of ultrasonic beam by the subcutaneous adipose tissue. According to our study, a 0.27 cutoff value of HSAI allows the separation between mild HS and moderate to severe HS with $87 \%$ accuracy, $76.6 \%$ sensitivity and $80.5 \%$ specificity. AUROC was 0.864 . One possible explanation for the lower HSAI statistical performances to assess HS in our patients cohort is the disproportion in the number of patients with moderatesevere HS (28.08\%) and mild HS (71.92\%). This disproportion has led to classification errors in the test group by the SVM classifier. On a more evenly distributed lot of patients in terms of HS, we expect the diagnosis accuracy to increase.

As regards the usage of a composite parameter (to perform a quantitative assesment on the echogenicity of hepatic parenchima loaded with fat in relation to other parenchyma with similar liver echogenicity), many previous studies have used the hepato-renal index for assessing HS. The sensitivity and specificity of HRI varied in different studies between 81.4 to $92.7 \%$ and $54-93 \%$ respectively. Wang [19] and Webb [18], using HRI for non-invasive quantification of the HS in relation to liver 
biopsy (gold standard), on groups of 175 and respectively 111 patients, reported Se of $84-90 \%$ and $\mathrm{Sp}$ of $85-93 \%$ for the prediction of moderate-severe HS ( $>25-30 \%$ ). SHRI cut-off value was 7 [19] and respectively 2.23 [18]. The major limitation of these approaches is the use of a reference system (kidney), whose echogenicity may also be altered by intrinsec illnesses. Therefore, the use of the kidney as a standard reference is improper because its echogenicity can be altered by chronic kidney diseases.

Even though the HRI sensitivity and specificity for the detection of moderate severe HS reported by various recent studies [18-22] are better (84-90\% Se, $85-93 \% \mathrm{Sp})$ compared to our results obtained by using CVL and HSAI parameters ( 76.6 to $81.3 \% \mathrm{Se}, 80.9$ to $89 \% \mathrm{Sp}$ ), we have to mention that these studies were conducted on smaller number of patients (88-175 pts) and not all of these studies used the histopathologic examination as validation method. For these reasons we believe that our findings could have even a better value as the above mentioned studies.

A limitation of our study is the imbalance between the two groups, namely the one with mild HS and the one with moderate-severe HS (164 vs 64 pts) which might justify some classification errors, especially in the group with S2-S3 steatosis. Moreover, using histopathology as a reference, it is possible that HS classification in the different severity classes might not be $100 \%$ accurate due to sampling errors, due to the fact that the Brunt score is semiquantitative and that the percentages close to the threshold between classes (e.g. 32 vs $34 \%$, 64 vs $66 \%$ ) are not properly labeled (thus automatically classifying the patient in another class of steatosis).

In conclusion, the proposed USICA method has proven to be an innovative, simple, accessible solution for the noninvasive assessment and quantification of HS, without being influenced by HF stage. The HSAI is a new, original parameter which facilitates quantification of liver steatosis even for mild degrees. It is reproducible and operator-independent and can be easily made available in routine clinical practice by integrating the USICA application to the US machine, thus improving the US diagnosis of HS by increasing Se and Sp specifically to identify mild HS $(<33 \%)$ which is difficult to assess by using conventional (visual) US examination.

\section{Conflict of interest: none}

Acknowledgments: The work was supported by an internal grant (1495/10/28.01.2014) financed by the "Iuliu Hatieganu" University of Medicine and Pharmacy Cluj-Napoca. This paper was published under the frame of the European Social Fund, Human Resources Development Operational Programme 2007-2013, project no. POSDRU/159/1.5/S/138776

\section{References}

1. Loomba R, Sanyal AJ. The global NAFLD epidemics. Nat Rev Gastroenterol Hepatol 2013; 10: 686-690.

2. Byrne CD, Targher G. NAFLD: A multisystem disease. J Hepatol 2015; 62: S47-S64.

3. Machado MV, Oliveira AG, Cortez-Pinto H. Hepatic steatosis in hepatitis B virus infected patients: meta-analysis of risk factors and comparison with hepatitis $\mathrm{C}$ infected patients. J Gastroenterol Hepatol 2011; 26: 1361-1367.

4. Powell EE, Jonsson JR, Clouston AD. Steatosis: co-factor in other liver diseases. Hepatology 2005; 42: 5-13.

5. Petta S, Camma C, Di Marco V, et al. Hepatic steatosis and insulin resistance are associated with severe fibrosis in patients with chronic hepatitis caused by HBV or HCV infection. Liver Int 2011; 31: 507-515.

6. Ates F, Yalniz M, Alan S. Impact of liver steatosis on reponse to pegylated interferon therapy in patients with chronic hepatitis B. World J Gastroenterol 2011; 17: 4517-4522.

7. Asselah T, Rubbia-Brandt L, Marcellin P, Negro F. Steatosis in chronic hepatitis $\mathrm{C}$ : why does it really matter? Gut 2006; 55: 123-130.

8. Bedosa P, Dargere D, Paradis V. Sampling variability of liver fibrosis in chronic hepatitis C. Hepatology 2003; 38 : 1449-1457.

9. Rockey DC, Caldwell SH, Goodman ZD, Nelson RC, Smith AD; American Association for the Study of Liver Diseases. Liver biopsy. Hepatology 2009; 49: 1017-1044.

10. Schwenzer NF, Springer F, Schraml C, Stefan N, Machann J, Schick F. Non-invasive assessment and quantification of liver steatosis by ultrasound, computed tomography and magnetic resonance. J Hepatol 2009; 51: 433-445.

11. Hernaez R, Lazo M, Bonekamp S, et al. Diagnostic accuracy and reliability of ultrasonography for the detection of fatty liver: a meta-analysis. Hepatology 2011; 54: 10821090.

12. Needleman DR, Kurtz AB, Rifkin MD, Cooper HS, Pasto $\mathrm{ME}$, Goldberg BB. Sonography of diffuse benign liver disease: accuracy of pattern recognition and grading. AJR Am J Roentgenol 1986; 146: 1011-1015.

13. Mojsilovic A, Popovic M, Markovic S, Krstić M. Characterization of visually similar diffuse diseases from B-scan liver images with nonseparable wavelet transform. IEEE Trans Med Imaging 1998; 17: 541-549.

14. Kadah YM, Farag AA, Zurada JM, Badawi AM, Youssef AM. A classification algorithms for quantitative tissue characterization of diffuse liver disease from ultrasound images. IEEE Trans Med Imaging 1996; 15: 466-478.

15. Lee CH, Choi JW, Kim KA, Seo TS, Lee JM, Park CM. Usefulness of standard deviation on the histogram of ultrasound as a quantitative value for hepatic parenchymal echo texture; preliminary study. Ultrasound Med Biol 2006; 32: 1817-1826.

16. Gaitini D, Lederman M, Baruch Y, et al. Computerised analysis of liver texture with correlation to needle biopsy. Ultraschall Med 2005; 26: 197-202.

17. Lupsor M, Badea R, Vicas C, et al. Detection of steatosis in chronic hepatitis $\mathrm{C}$, based on the evaluation of the at- 
tenuation coefficient computed on ultrasound image. Med Ultrason 2008; 10: 13-20.

18. Webb M, Yeshua H, Zelber-Sagi S, et al. Diagnostic value of a computerized hepatorenal index for sonographic quantification of liver steatosis. AJR Am J Roentgenol 2009; 192: 909-914.

19. Wang JH, Hung CH, Kuo FY, et al. Ultrasonographic quantification of hepatic-renal echogenicity difference in hepatic steatosis. Dig Dis Sci 2013; 58: 2993-3000.

20. Xia MF, Yan MH, He WY, et al. Standardized ultrasound hepatic/renal ratio and hepatic attenuation rate to quantify liver fat content: An improvement method. Obesity 2012; 20: 444-452.

21. Borges VF, Diniz AL, Cotrim HP, Rocha HL, Andrade NB. Sonographic hepatorenal ratio: a noninvasive method to diagnose nonalcoholic steatosis. J Clin Ultrasound 2013; 41: $18-25$.

22. Zhang B, Ding F, Chen T, Xia LH, Qian J, Lv GY. Ultrasound hepatic/renal ratio and hepatic attenuation rate for quantifying liver fat content. World J Gastroenterol 2014; 20: 17985-17992.

23. Nagy G, Gordan M, Vlaicu A, Mircea PA, Crisan D, Valean $\mathrm{S}$. Non-invasive evaluation of hepatic steatosis by ultrasound image analysis with simple brightness features and support vector machines. Acta Electrotehnica 2007; 48: 217-222.

24. Brunt EM, Janney CG, Di Bisceglie AM, NeuschwanderTetri BA, Bacon BR. Nonalcoholic steatohepatitis: A proposal for grading and staging the histological lesions. Am J Gastroenterol 1999; 94: 2467-2474.

25. Cristianini N, Shawe-Taylor J. An Introduction to Support Vector Machines. Cambridge University Press, Cambridge, UK, 2000.

26. Vapnik VN. Statistical Learning Theory. J. Wiley, NY, 1998.

27. Joy D, Thava VR, Scott BB. Diagnosis of fatty liver disease: is biopsy necessary? Eur J Gastroenterol Hepatol 2003; 15: 539-543. 